\title{
Chemistry of Materials
}

Morphological and Kinetic Studies on Hexagonal Tungstates Alexej Michailovski, Ragnar Kiebach, Wolfgang Bensch, Jan-Dierk Grunwaldt, Alfons Baiker, Sridhar Komarneni, and Greta R. Patzke*

\section{Supporting Information}

\section{Table of Contents}

Table S1 Synthesis of HTB-/P alkali tungstates with soft chemistry methods

Literature: Table S1

Table S2 The rate equations for solid state reactions reported in literature

Figure S1 SEM of lithium tungstate (standard reaction conditions) 
Table S1. Synthesis of HTB-/P alkali tungstates(VI) with soft chemistry methods

\begin{tabular}{|c|c|c|c|c|}
\hline Compound & Type & Synthesis & Precursor & Lit. \\
\hline $\begin{array}{l}(1.03-1.32) \mathrm{Li}_{2} \mathrm{O} \cdot \mathrm{WO}_{3} \cdot \\
(0.74-0.99) \mathrm{H}_{2} \mathrm{O}\end{array}$ & $\mathrm{O}$ & solution & $\mathrm{M}_{2} \mathrm{WO}_{4}, \mathrm{HCl}$ or $\mathrm{HNO}_{3}, 100^{\circ} \mathrm{C}$ & [1] \\
\hline $\mathrm{Li}_{0.20} \mathrm{WO}_{3.10} \cdot 0.5 \mathrm{H}_{2} \mathrm{O}$ & HTB & hydrothermal & $\mathrm{M}_{2} \mathrm{WO}_{4}, \mathrm{HCl}, 155^{\circ} \mathrm{C}$ & [2] \\
\hline $\begin{array}{l}\mathrm{Li}_{(0.10-0.42)} \mathrm{WO}_{(3.05-3.21)^{\circ}} \\
\quad 0.56-0.88 \mathrm{H}_{2} \mathrm{O}\end{array}$ & HTB & solution & $\begin{array}{l}\mathrm{Li}_{2} \mathrm{WO}_{4}, \mathrm{HCl} \\
125-200{ }^{\circ} \mathrm{C}\end{array}$ & [3] \\
\hline $\begin{array}{c}(0.08-0.14) \mathrm{Na}_{2} \mathrm{O} \cdot \mathrm{WO}_{3} \cdot \\
(0.67-0.85) \mathrm{H}_{2} \mathrm{O}\end{array}$ & HTB & solution & $\mathrm{M}_{2} \mathrm{WO}_{4}, \mathrm{HCl}$ or $\mathrm{HNO}_{3}, 100^{\circ} \mathrm{C}$ & [1] \\
\hline $\mathrm{Na}_{0.26} \mathrm{WO}_{3.13} \cdot 0.5 \mathrm{H}_{2} \mathrm{O}$ & HTB & hydrothermal & $\mathrm{M}_{2} \mathrm{WO}_{4}, \mathrm{HCl}, 155^{\circ} \mathrm{C}$ & [2] \\
\hline $\mathrm{Na}_{0.55} \mathrm{WO}_{3.275}$ & $\mathrm{P}$ & hydrothermal & $\mathrm{M}_{2} \mathrm{WO}_{4}, \mathrm{HCl}, 155^{\circ} \mathrm{C}$ & {$[2]$} \\
\hline 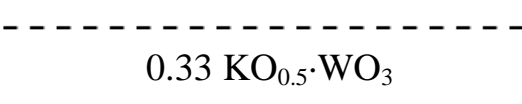 & $\begin{array}{ll}--- \\
\text { HTB }\end{array}$ & $\begin{array}{l}------ \\
\text { solution }\end{array}$ & $\begin{array}{c}\text { M-IPA and MCl, RT }\end{array}$ & $\begin{array}{l}---- \\
{[4]}\end{array}$ \\
\hline $\begin{array}{l}(0.07-0.11) \mathrm{K}_{2} \mathrm{O} \cdot \mathrm{WO}_{3} \cdot \\
\quad(0.49-0.67) \mathrm{H}_{2} \mathrm{O}\end{array}$ & НTB & solution & $\mathrm{M}_{2} \mathrm{WO}_{4}, \mathrm{HCl}$ or $\mathrm{HNO}_{3}, 100^{\circ} \mathrm{C}$ & {$[1]$} \\
\hline $\mathrm{K}_{\mathrm{x}} \mathrm{W}_{1-\mathrm{y} / 6} \mathrm{O}_{3}$ & НTB & heating & $\mathrm{K}$-IPA, air, $500{ }^{\circ} \mathrm{C}$ & [5] \\
\hline $0.23 \mathrm{RbO}_{0.5} \cdot \mathrm{WO}_{3}$ & HTB & solution & M-IPA and MCl, RT & [4] \\
\hline $\mathrm{Rb}_{\mathrm{x}} \mathrm{WO}_{3+\mathrm{x} / 2}$ & $\mathrm{P}$ & hydrothermal & $\mathrm{M}_{2} \mathrm{WO}_{4}, \mathrm{HCl}, 155^{\circ} \mathrm{C}$ & [2] \\
\hline $\mathrm{Rb}_{1.1} \mathrm{~W}_{1.65} \mathrm{O}_{5.5}$ & $\mathrm{P}$ & solid state & $\mathrm{h}-\mathrm{WO}_{3}, \mathrm{Rb}_{2} \mathrm{CO}_{3}, 300{ }^{\circ} \mathrm{C}$ & [6] \\
\hline $\mathrm{Cs}_{\mathrm{x}} \mathrm{W}_{1-\mathrm{y} / 6} \mathrm{O}_{3}(\mathrm{x}=0.3)$ & HTB & solution & Cs-poor IPA and CsCl, RT & [7] \\
\hline $\mathrm{Cs}_{\mathrm{x}} \mathrm{W}_{1-\mathrm{y} / 6} \mathrm{O}_{3}$ & $\mathrm{P}$ & solution & Cs-rich IPA and CsCl & [7] \\
\hline $0.45 \mathrm{CsO}_{0.5} \cdot \mathrm{WO}_{3}$ & $\mathrm{P}$ & solution & M-IPA and MCl, RT & [4] \\
\hline$\left(\mathrm{Cs}_{2} \mathrm{O}\right)_{\mathrm{x}} \mathrm{W}_{2} \mathrm{O}_{6}(0.3<\mathrm{x}<0.5)$ & $\mathrm{P}$ & solution & $\mathrm{Na}_{2} \mathrm{WO}_{4} \cdot 2 \mathrm{H}_{2} \mathrm{O}, \mathrm{HCl}, \mathrm{Cs}_{2} \mathrm{CO}_{3}, 90^{\circ} \mathrm{C}$ & {$[8]$} \\
\hline $\mathrm{Cs}_{\mathrm{x}} \mathrm{WO}_{3+\mathrm{x} / 2}$ & $\mathrm{P}$ & hydrothermal & $\mathrm{M}_{2} \mathrm{WO}_{4}, \mathrm{HCl}, 155^{\circ} \mathrm{C}$ & {$[2]$} \\
\hline $\mathrm{Cs}_{\mathrm{x}} \mathrm{WO}_{3+\mathrm{y}}$ & $P$ & ball-milling & $\mathrm{WO}_{3}, \mathrm{Cs}_{2} \mathrm{CO}_{3}$ & [1] \\
\hline $\mathrm{Cs}_{1.1} \mathrm{~W}_{1.65} \mathrm{O}_{5.5}$ & $\mathrm{P}$ & solid state & $\mathrm{h}-\mathrm{WO}_{3}, \mathrm{Cs}_{2} \mathrm{CO}_{3}, 300^{\circ} \mathrm{C}$ & {$[6]$} \\
\hline $\begin{array}{c}\left.(0.04-0.073)\left\{\left(\mathrm{NH}_{4}\right)_{2} \mathrm{O}\right)\right\} \cdot \mathrm{WO}_{3} \\
(0.38-0.82) \mathrm{H}_{2} \mathrm{O}\end{array}$ & HTB & solution & $\mathrm{M}_{2} \mathrm{WO}_{4}, \mathrm{HCl}$ or $\mathrm{HNO}_{3}, 100{ }^{\circ} \mathrm{C}$ & {$[1]$} \\
\hline$\left(\mathrm{NH}_{4}\right)_{0.20} \mathrm{WO}_{3.10}$ & HTB & hydrothermal & $\mathrm{M}_{2} \mathrm{WO}_{4}, \mathrm{HCl}, 155^{\circ} \mathrm{C}$ & [2] \\
\hline $\mathrm{Ba}_{\mathrm{x}} \mathrm{W}_{1-\mathrm{y} / 3} \mathrm{O}_{3}$ & 2D-P & solution & M-IPA and $\mathrm{MCl}_{2}, \mathrm{RT}$ & [4] \\
\hline $\mathrm{Tl}_{\mathrm{x}} \mathrm{W}_{1-\mathrm{y} / 6} \mathrm{O}_{3}$ & 2D-P & solution & $\mathrm{M}-\mathrm{IPA}$ and $\mathrm{MCl}_{2}, \mathrm{RT}$ & {$[4]$} \\
\hline $\mathrm{Pb}_{\mathrm{x}} \mathrm{W}_{1-\mathrm{y} / 3} \mathrm{O}_{3}$ & 2D-P & solution & M-IPA and $\mathrm{MCl}_{2}, \mathrm{RT}$ & [4] \\
\hline
\end{tabular}




\section{Literature: Table S1}

[1] Kumagai, N.; Matsuura, Y.; Umetzu, Y.; Tanno, K. Solid State Ionics 1992, 53 56, 324.

[2] Reis, K. P.; Ramanan, A.; Whittingham, M. S. J. Solid State Chem. 1992, 96, 31.

[3] Yu, A.; Kumagai, N.; Liu, Z.; Lee, Y. J. Solid State Electrochem. 1998, 2, 394.

[4] Kudo, T.; Oi, J.; Kishimoto, A.; Hiratani, M. Mat. Res. Bull. 1991, 26, 779.

[5] Tsuyumoto, I.; Kishimoto, A.; Kudo, T. Solid State Ionics 1993, 59, 211.

[6] Driouiche, A.; Abraham, F.; Touboul, M.; Figlarz, M. Mat. Res. Bull. 1991, 26, 901.

[7] Oi, J.; Kishimoto, A.; Kudo, T. J. Solid State Chem. 1993, 103, 176.

[8] Coucou, A.; Figlarz, M. Solid State Ionics 1988, 28 - 30, 1762. 
Table S2. The rate equations for solid state reactions reported in literature ${ }^{[1-3]}$

\begin{tabular}{|c|c|c|c|}
\hline \multicolumn{2}{|c|}{ Growth Model } & Rate Equation $f(\alpha)=\mathbf{k t}$ & $m$ \\
\hline \multicolumn{4}{|c|}{ Diffusion controlled } \\
\hline $\mathrm{D}_{1}(\alpha)$ & & $\alpha^{2}=0.25\left(t / t_{0.5}\right)$ & 0.62 \\
\hline $\mathrm{D}_{2}(\alpha)$ & & $(1-\alpha) \ln (1-\alpha)+\alpha=0.1534\left(\mathrm{t} / \mathrm{t}_{0.5}\right)$ & 0.57 \\
\hline $\mathrm{D}_{3}(\alpha)$ & [Jander] & {$\left[1-(1-\alpha)^{1 / 3}\right]^{2}=0.0425\left(t / t_{0.5}\right)$} & 0.54 \\
\hline $\mathrm{D}_{4}(\alpha)$ & [Ginstling-Brounshtein] & $1-2 \alpha / 3-(1-\alpha)^{2 / 3}=0.0367\left(\mathrm{t} / \mathrm{t}_{0.5}\right)$ & 0.57 \\
\hline \multicolumn{4}{|c|}{ Phase-boundary controlled } \\
\hline $\mathrm{R}_{2}(\alpha)$ & & $1-(1-\alpha)^{1 / 2}=0.2929\left(t / t_{0.5}\right)$ & 1.11 \\
\hline $\mathrm{R}_{3}(\alpha)$ & & $1-(1-\alpha)^{1 / 3}=0.2063\left(t / t_{0.5}\right)$ & 1.07 \\
\hline \multicolumn{4}{|c|}{ First order } \\
\hline $\mathrm{F} 1(\alpha)$ & & {$[-\ln (1-\alpha)]=0.6931\left(\mathrm{t} / \mathrm{t}_{0.5}\right)$} & 1.00 \\
\hline \multicolumn{4}{|c|}{ Nucleation [Avrami-Erovéef] } \\
\hline $\mathrm{A} 2(\alpha)$ & & {$[-\ln (1-\alpha)]^{1 / 2}=0.8326\left(t / t_{0.5}\right)$} & 2.00 \\
\hline $\mathrm{A} 3(\alpha)$ & & {$[-\ln (1-\alpha)]^{1 / 3}=0.885\left(\mathrm{t} / \mathrm{t}_{0.5}\right)$} & 3.00 \\
\hline
\end{tabular}

\section{References}

[1] Brown, P. W.; Pommersheim, J.; Frohnsdorff, G. Cem. Concr. Res. 1985, 15, 35.

[2] Thomas, J. J.; Jennings, H. M. Chem. Mater. 1999, 11, 1907.

[3] (a) Avrami, M. J. Chem. Phys. 1939, 7, 1103. (b) Avrami, M. J. Chem. Phys. 1940, 8, 212. (c) Avrami, M. J. Chem. Phys. 1941, 9, 177. 
Figure S1. Representative SEM images of lithium tungstates synthesized under standard conditions.

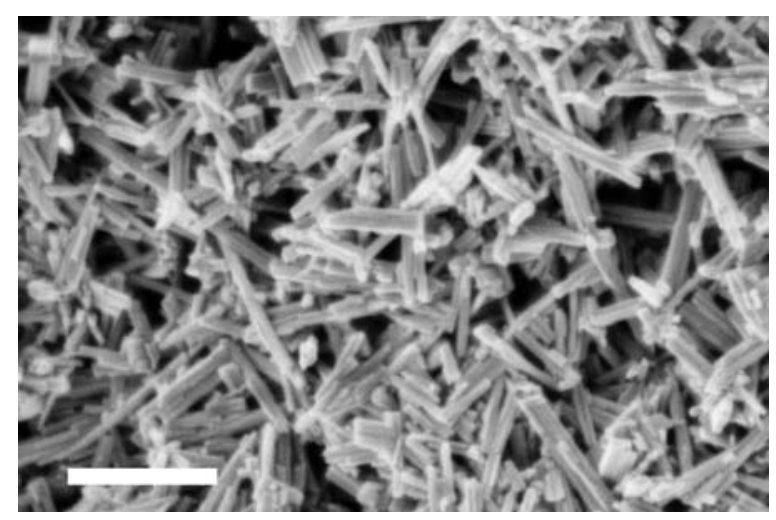

Figure S2. Representative SEM images of Na-HTB tungstate rods synthesized under standard conditions (a) and of K-HTB tungstate rods synthesized in the $\mathrm{KCl} / \mathrm{AMT} / \mathrm{H}_{2} \mathrm{O}$ system with AMT dilution $\left(\mathrm{c}(\mathrm{W})=0.07 \mathrm{M}\right.$ in $\left.\mathrm{H}_{2} \mathrm{O}\right)(\mathrm{b})$.
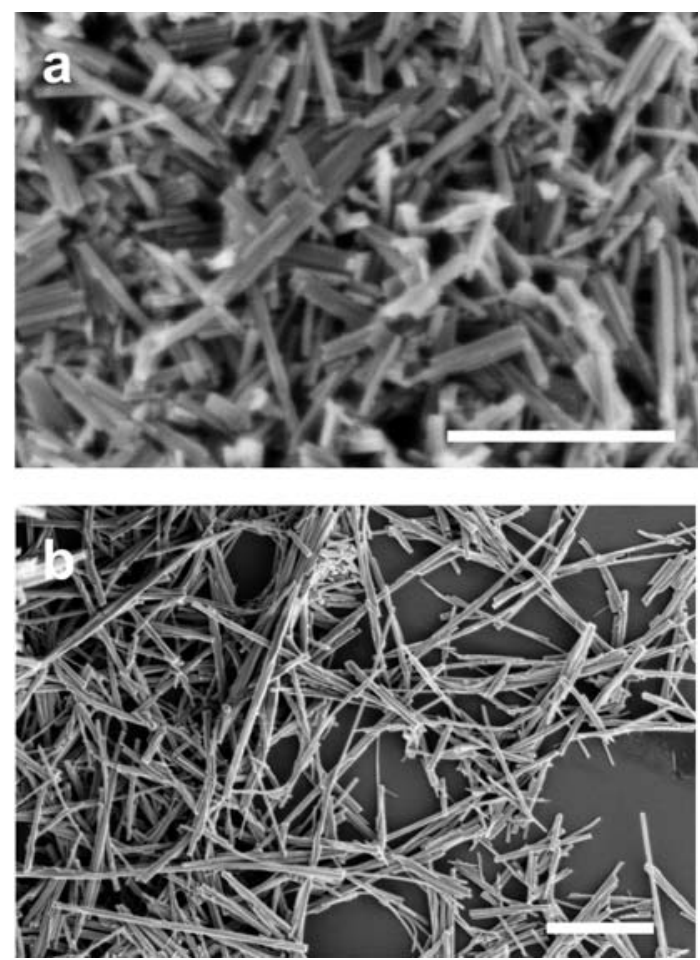
Figure S3. Representative SEM images (scale bar $=1 \mu \mathrm{m}$ ) of Na-HTB tungstate rods $(\mathrm{c}(\mathrm{W})=0.07 \mathrm{M})$ : (a) after $4 \mathrm{~h}$ of hydrothermal treatment, (b) after $18 \mathrm{~d}$ of hydrothermal treatment.
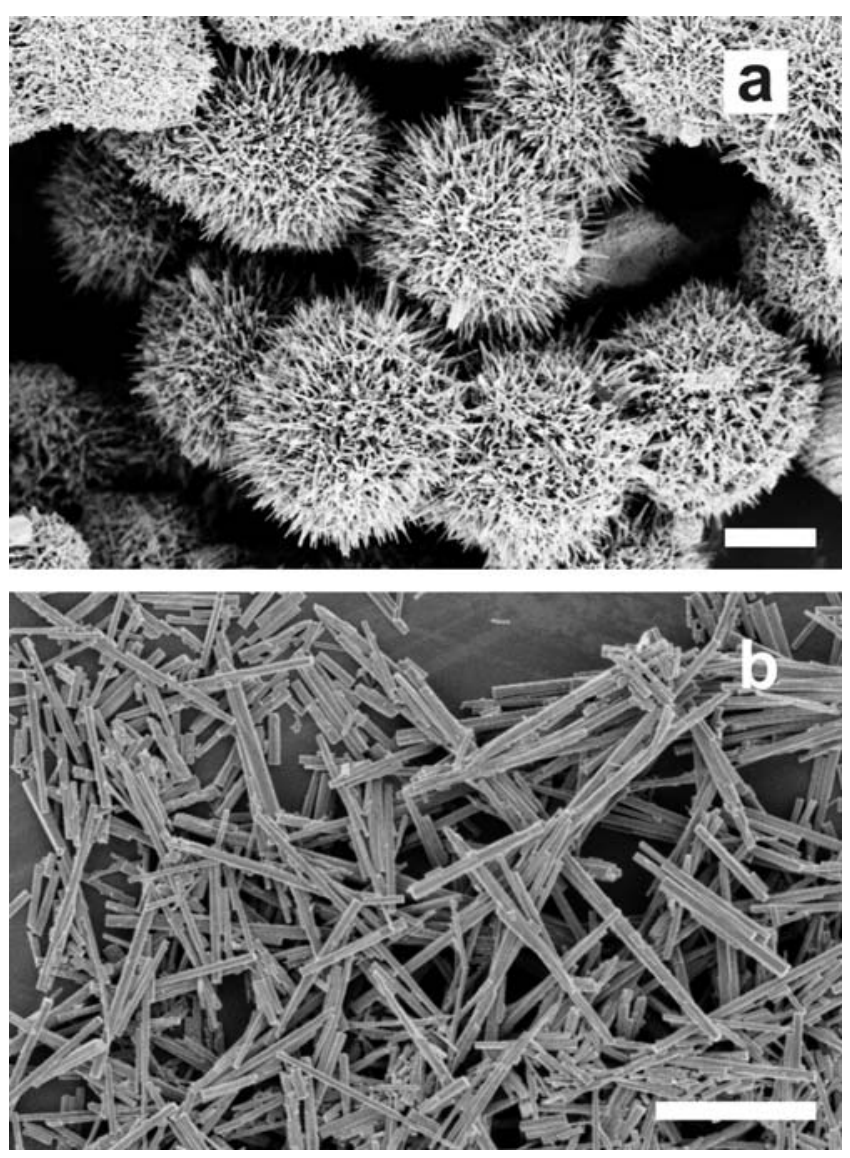
Figure S4. Representative SEM images of nanostructured tungstates after $18 \mathrm{~d}$ of hydrothermal treatment $(\mathrm{c}(\mathrm{W})=0.007 \mathrm{M})$ : (a, b) Li-O tungstates, (c, d) Cs-HTB tungstates.
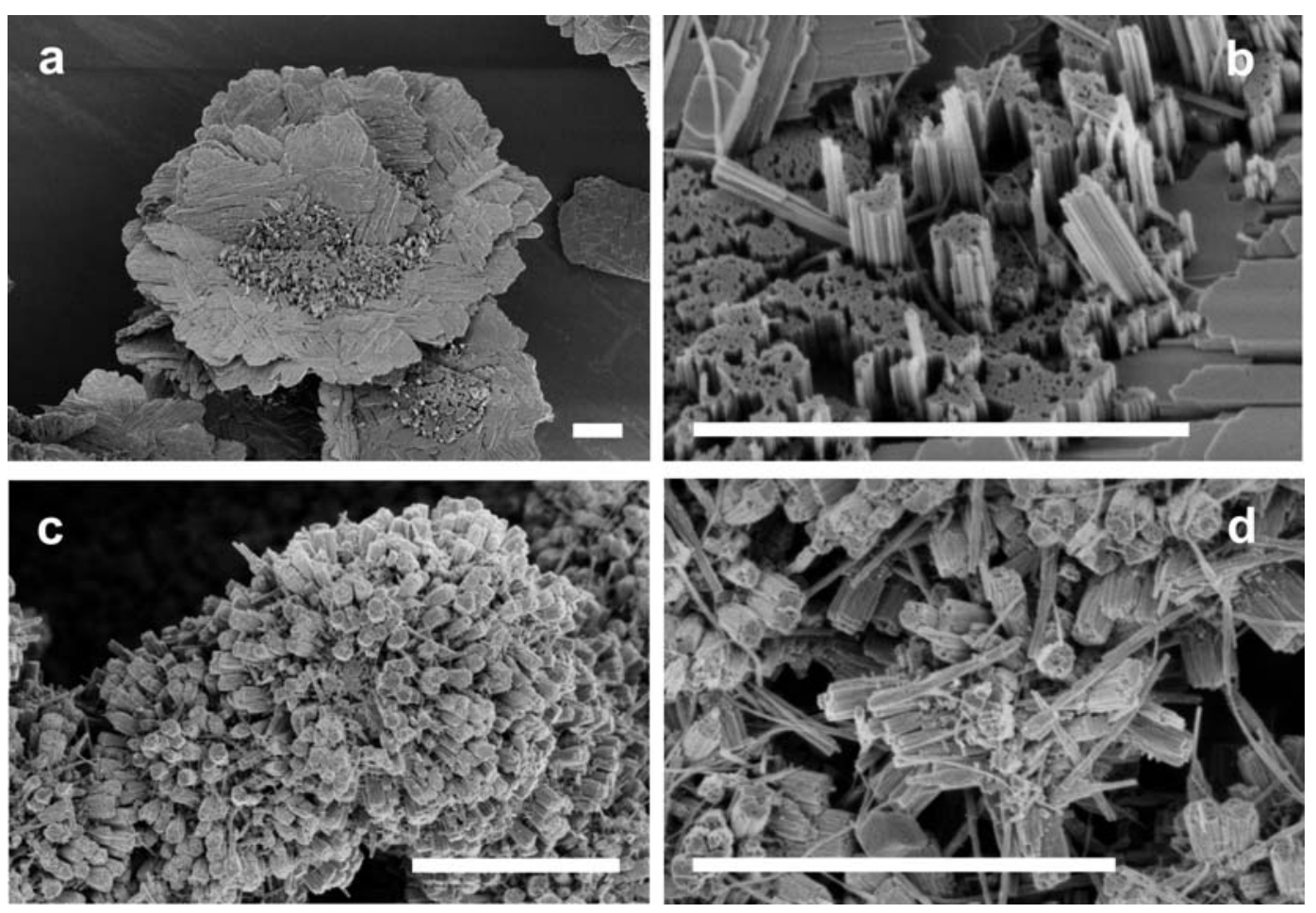